\title{
Qualität sichtbar machen: Ergebnisqualität und Evaluationsinstrumente für Freiwilligendienste
}

\author{
Ute Elisabeth Volkmann \\ Dipl. Kulturpäd., M.A. Kultur- und Erziehungswissenschaften \\ Agentur für Qualität in Freiwilligendiensten (Quifd) | Projektleiterin \\ utevolkmann@berlin.de
}

Freiwilligendienste, verstanden als Bildungsdienste, werden in ihren Ergebnissen und Wirkungen kontrovers diskutiert. Grundsätzlich wird in dieser Diskussion davon ausgegangen, dass Freiwilligendienste Ergebnisse und Wirkungen auf unterschiedlichen Ebenen hinterlassen und abgesehen von den Selbstveränderungen der Freiwilligen, auch Veränderungen auf der Meso-Ebene der Organisationen und Einsatzstellen sowie auf gesamtgesellschaftlicher Ebene bewirken können. Quifd, die Agentur für Qualität in Freiwilligendiensten, greift das Interesse an wirkungsorientierter Betrachtung der Freiwilligendienste in dem Projekt zur ,Entwicklung von Standards für Ergebnisqualität und von Evaluationsinstrumenten für Freiwilligendienste" auf. Mit dem Ziel, Trägerorganisationen in ihren Qualitätsbemühungen zu unterstützen wurden theoriegestützte Fragebögen für Längsschnitterhebungen, Wirkungshypothesen und Kann-Standards ${ }^{I}$ für die Ergebnisqualität entwickelt. Dieser Beitrag stellt das Projekt mit seinen Resultaten sowie die im Rahmen des Fachtags „Ergebnisqualität von Freiwilligendiensten erkennen und optimieren" am 9. September 2013 in Berlin

${ }^{1}$ In Zusammenarbeit mit Wissenschaft und Praxis hat Quifd Qualitätsstandards für Einsatzstellen, Träger- und Entsendeorganisationen erarbeitet. Quifd unterscheidet dabei zwischen Soll- und Kann-Standards. Während die Erfüllung der SollStandards die Grundlage darstellt für die Zertifizierung mit dem Quifd Gütesiegel, stellen die Kann-Standards keine obligatorische Voraussetzung der Zertifizierung dar. Sie dienen den Organisationen zunächst lediglich als Orientierung für weitere Qualitätsmerkmale ihrer Arbeit. 
kritisch geführte Diskussion um Nutzen und Grenzen von Ergebnisqualitätsmessung vor.

Schlagworte: Wirkungserwartungen, Ergebnisqualität, trägerunspezifisches Evaluationsinstrument, Ergebnisqualitätsstandards, Wirkungshypothesen

\section{Vorïberlegungen}

Im Widerstreit von Ergebnisoffenheit und Standardisierung stellen die hier vorgestellten Projektresultate eine erste trägerübergreifende Möglichkeit dar, über die erhobenen individuellen oder aggregierten Ergebnisse, Hinweise auf die Qualität der freiwilligendienstimmanenten Strukturen und Prozesse zu erhalten und damit die Qualitätsentwicklung der Freiwilligendienste methodisch voranzutreiben. Aus Kapazitätsgründen liegt mit der Untersuchung der Ergebnisqualität bei den Freiwilligen, der Fokus des Projekts zunächst auf der mikrosoziologischen Perspektive nicht aber auf dem Mikroumfeld der Freiwilligen in den Einsatzstellen.

Die Heterogenität der unterschiedlichen Freiwilligendienstformate ebenso wie die Diversität der Trägerorganisationen reflektierend, liegt es nicht fern zu antizipieren, dass die Erwartungen an die Ergebnisse von Freiwilligendiensten alleine auf der Mikro-Ebene der Freiwilligen höchst unterschiedlich ausfallen. Auch die Motivlagen der unterschiedlichen Akteure in den Freiwilligendiensten, Ergebnisse systematisch zu erheben, sind dementsprechend ausdifferenziert. Daraus ergibt sich in der praktischen und politischen Landschaft der Jugendfreiwilligendienste eine inhaltliche Kontroverse zwischen konkreten Entwicklungserwartungen und höchst subjektiven Veränderungen ebenso wie eine methodische Debatte zwischen quantitativen und qualitativen Ansätzen der Ergebniserhebung.

\section{Die Kontroverse: Wirkungserwartungen versus Ergebnisoffenheit}

Mit einer Anzahl von jährlich ca. 90.000 Freiwilligen in den Inlandsdiensten und ca. 5000 Freiwilligen in den grenzüberschreitenden Diensten stehen die Freiwilligendienste im Zentrum der Diskussion um Wirkungen und Ergebnisse formal geregelten Engagements. Erwartungen an den individuellen und gesellschaftlichen Nutzen stehen dabei neben der Betonung des Eigensinns 
der Freiwilligendienste. So werden die Freiwilligendienste einerseits diskutiert als Moratorium, als unverzweckter Freiraum und Ort der Entschleunigung, bei dem die Freiwilligen, frei von Leistungsdruck und ökonomischen Effizienzerwartungen, (Aus-)Zeit und Gelegenheit zur Selbstentfaltung erhalten. Da, wo Bildungsbiografien zeitlich immer enger gestrickt und auf formale Bildungsabschlüsse fokussiert sind, sollen die Freiwilligendienste aus dieser Perspektive heraus der Ort sein, der Raum für Überraschungen bereithält und prägende, aber höchst subjektive Erlebnisse, Begegnungen und Erfahrungen und damit individuelle Wirkungen und Ergebnisse eröffnet. Andererseits werden die Freiwilligendienste diskutiert als ein Format der sozialen Bildung, als Ort des informellen und non-formalen Lernens, wo die Freiwilligen im Ergebnis unterschiedliche Kompetenzen entwickeln, sich beruflich orientieren und gesellschaftliche Partizipationsräume kennenlernen sollen.

\subsection{Die Interessen: Wissensdurst versus Befragungsfrust}

Spielen die Freiwilligendienste als Gegenstand wissenschaftlicher und unabhängiger universitärer Untersuchungen zumindest in Deutschland bislang keine zentrale Rolle, so formiert sich doch auf politischer und praktischer Ebene der Wunsch, den Wirkungen und Ergebnissen von Freiwilligendiensten systematisch nachzugehen. Davon zeugt die vom Bundesfamilienministerium (BMFSFJ) beauftragte groß angelegte Evaluation des Freiwilligen Sozialen und Ökologischen Jahres (FSJ/FÖJ) und des Bundesfreiwilligendienstes (BFD) ebenso wie eine Vielzahl individueller Befragungen der Trägerorganisationen mit wirkungsorientierten Frageanteilen. In Zeiten outcome-gesteuerter Finanzierung, Kontingentierung und Mittelbewilligung mag eine Motivation dafür die Legitimation und der Nachweis der qualitätvollen Leistung der Träger sein. Viele mögen ein ebenso intrinsisches Interesse haben, nachzuvollziehen und im Sinne ihrer Qualitätsentwicklung zu evaluieren, welche Wirkungen ihre Dienste bei den Freiwilligen hinterlassen. Von dem aktiven Anliegen der Trägerorganisationen, die Ergebnisse und Wirkungen ihrer Freiwilligendienste $\mathrm{zu}$ untersuchen und $\mathrm{zu}$ verbessern, zeugten in der Vergangenheit diverse Veranstaltungen im Portfolio von Quifd, wie die großformatige Tagung zu „Qualität und Wirkung von Freiwilligendiensten“ im September 2009, Veranstaltungen zu „Wirkung und Wirkungserfassung in Freiwilligendiensten" im Februar 2010/2011 sowie Weiterbildungen zur „Fragebogenentwicklung als Metho- 
de der Selbstevaluation“"im Oktober 2012. Das Interesse, das all diesen Veranstaltungen zugrunde lag, lässt sich zusammenfassen als die Frage danach, „Wer, wie oder was wirkt in den Freiwilligendiensten und wie lässt sich das messen?". Gleichzeitig impliziert diese Frage die inhaltlichen und methodischen Herausforderungen der Ergebnismessung und Wirkungsforschung in den Freiwilligendiensten, die sich neben Zuordnungslücken zwischen Erleben und Wirken und Validitätsaspekten auch auf die methodische und moralische Frage, „Wie ist der Wissensdurst der einen mit dem Befragungsfrust der anderen zu vereinbaren?" bezieht, wenn die Freiwilligen im Verlauf des Dienstes gebeten werden fünf oder mehr verschiedene Fragebögen auszufüllen, um nach jedem Seminar und gegen Ende ihres Dienstes den Einsatzstellen, Trägerorganisationen und anderen Akteuren Feedback zu geben.

\section{Das Quifd-Projekt: Ergebnisqualität}

Mit dem Handlungsauftrag von Quifd, der Agentur für Qualität in Freiwilligendiensten, Trägerorganisationen von Freiwilligendiensten in der Qualitätsentwicklung zu unterstützen, besteht ein zentrales Anliegen der Agentur darin, die unterschiedlichen Interessen der Träger an Ergebnissen und Wirkungen in den fachlich-methodischen Qualitätsdiskurs der Freiwilligendienste einzubringen und methodische Ansätze zur Qualitätsentwicklung bereitzustellen. Bislang liegt der Fokus von Quifd auf der qualitativen Weiterentwicklung der Strukturen und Prozesse der Freiwilligendienste. Qualitätsstandards für freiwilligendienstimmanente Strukturen und Prozesse bieten den Trägerorganisationen dabei handlungsorientierte Vorgaben für die Ausgestaltung verlässlicher und qualitätvoller Rahmenbedingungen für freiwilliges Engagement. Ein Verfahren der Selbst- und Fremdevaluation sowie der Zertifizierung mit einem Qualitätssiegel bieten auf Träger- und Einsatzstellenebene methodische Unterstützung und Anreiz, sich systematisch mit der Qualität der eigenen Strukturen und Prozesse auseinanderzusetzen und diese zu verbessern. Als Labor für die Entwicklung neuer Qualitätsinstrumente ist Quifd darüber hinaus seit vielen Jahren daran interessiert, das bestehende Qualitätsmanagementsystem für Freiwilligendienste um die Ergebnisqualität zu ergänzen, d. h. zu vervollständigen. Mit dem übergeordneten Projektziel, über die Erhebung der Ergebnisqualität einen Beitrag zur Qualitätsentwicklung der Freiwilligendienste zu leisten und den Trägerorga- 
nisationen von Freiwilligendiensten methodische und analytische Unterstützung bieten zu können, hat Quifd sich den inhaltlichen und methodischen Herausforderung der Ergebniserfassung nun aktiv gestellt. Gefördert von der Robert Bosch Stiftung führte Quifd unter dem Titel „Entwicklung von Standards für Ergebnisqualität und von Evaluationsinstrumenten für Freiwilligendienste“ von Februar 2012 bis September 2013 ein Projekt zur Entwicklung von Evaluationsinstrumenten für Ergebnisse von Freiwilligendiensten durch, das im Folgenden mit seinen Ausgangsthesen, zentralen Leitfragen, in seiner theoretischen Fundierung, seinen Meilensteinen und Resultaten vorgestellt wird. Die Zielstellung des Projekts bestand in der Entwicklung eines Evaluationsinstruments, mit dem die Träger von Freiwilligendiensten die Ergebnisse ihrer Dienste, die im Folgenden ausführlich erläutert werden, im Rahmen einer Vorher-Nachher-Befragung der Freiwilligen eruieren und damit Hinweise auf die Qualität ihrer Prozesse und Strukturen ableiten können.

Mit der abschließenden Präsentation und Diskussion des Projekts anlässlich des erwähnten Fachtags im September 2013 wurde nicht nur der Fragebogen, sondern auch mögliche Ergebnisse von Freiwilligendiensten und methodische Ansätze ihrer Messbarkeit, in ihren Chancen und Grenzen, ihren Vorteilen und Risiken, ihre politischen und praktischen Implikationen von TrägerInnen, PolitikvertreterInnen, WissenschaftlerInnen und MultiplikatorInnen ausführlich debattiert. Im Fokus stand mit dem trägerübergreifenden, integrier- und anpassbaren Befragungsinstrument ein Novum in den methodischen Qualitätsbemühungen in den Freiwilligendiensten.

\section{Meilensteine in Richtung Messbarkeit}

Gerade da, wo neben praktischen Widerständen auch Vorbehalte aus der Wissenschaft im Raum stehen, die die methodischen Hürden und Zuordnungslücken beschreiben, um Wirkungen und Ergebnisse bei den Freiwilligen kausal auf die Freiwilligendienste zurückzuführen, galt es zu Beginn der Projektentwicklung, genau abzugrenzen, welche Ergebnisebene der Freiwilligendienste im Zentrum des Projekts steht. So werden Ergebnisse hier verstanden als die Veränderungen, die durch den Prozess und die Strukturen des Freiwilligendienstes stimuliert und bewirkt werden können. Ergebnisse werden darüber hinaus abgebildet anhand der Zufriedenheit der Freiwilligen mit den dienstimmanenten Strukturen und Prozessen. Die Veränderungen 
und die Zufriedenheit werden im Rahmen des Projekts ausschließlich auf der Mikroebene der Freiwilligen und auf der zeitlichen Ebene als Veränderungen unmittelbar nach dem Freiwilligendienst fokussiert. Klassische Wirkungsketten in ihrer eher abstrakten Terminologie des Input, Output und Outcome werden im Sinne der Praxisorientierung übersetzt als die Aktivitäten und Ressourcen der Träger (Input), die wiederum in bestimmte Prozesse und Strukturen (Output) überführt werden und damit die Rahmenbedingungen der stattgefundenen Prozesse und des individuellen Erlebens der Freiwilligen konstituieren. Im Sinne der Qualitätsentwicklung sind es eben diese Prozesse der sozialen Interaktion mit den unterschiedlichen beteiligten Akteuren der Trägerorganisation und der Einsatzstelle und die dadurch initiierten Prozesse des Erlebens, Begegnens und Erfahrens der Freiwilligen, die neben vielfältigen anderen Faktoren am Ende des Freiwilligendienstes zu Veränderungen und Ergebnissen (Outcome) bei den Freiwilligen und damit auch zu einer guten Qualität der Dienste führen können. Die Impact-Ebene die so genannten langfristigen Wirkungen - ist hingegen nicht Gegenstand dieses Projektes gewesen.

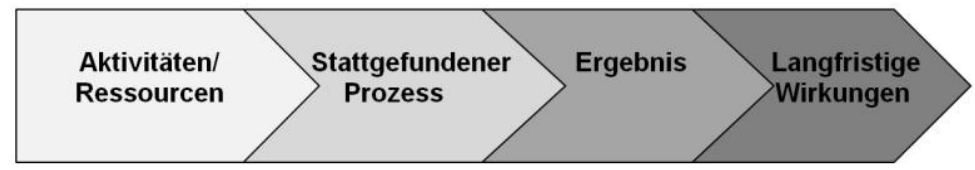

Abb. 1: Modell der Wirkungs- und Ermöglichungshypothesen

Quelle: Eigene Darstellung

\section{Ausgangsthesen und Leitfragen}

Auf diesen funktionalistischen Überlegungen aufbauend lautet die zentrale Ausgangsthese des Projekts Freiwilligendienste wirken. Sie bewirken Veränderungen bei den Freiwilligen. Darüber hinaus liegt dem Projekt die These zugrunde, dass diese Veränderungen, die als Ergebnisse unmittelbar nach dem Dienst erhoben werden, durch Strukturen und Prozesse bei den Trägern und den Einsatzstellen beeinflussbar sind. Daraus folgen die forschungsleitenden Fragen des Projekts, zunächst nach den konkreten Veränderungen und Ergebnissen, die auf der Mikroebene der Freiwilligen im Rahmen der unterschiedlichen Jugendfreiwilligendienstformate realistisch sind. Hier war eine Auswahl der aus Sicht der mitwirkenden TrägervertreterInnen wichtigs- 
ten Ergebnisse von Freiwilligendiensten notwendig, wohlwissend, dass die Freiwilligendienste noch vielfältigere (sehr individuelle) Veränderungen erzielen. Nachdem die Ergebnisdimensionen auf der Mikroebene der Freiwilligen ausgewählt wurden, stellten sich die Fragen, auf welche Strukturen und Prozesse diese Veränderungen zurückzuführen sind und wie sich sowohl die Prozesse und Strukturen als auch die Veränderungen anhand methodisch valider Fragestellungen operationalisieren lassen.

Als konkretes Projektergebnis wurde somit ein Evaluationsinstrument, sprich ein Fragebogen zur Vorher-Nachher-Befragung der Freiwilligen sowie die Entwicklung von Wirkungshypothesen anvisiert. Eine Befragung der Freiwilligen im Längsschnittdesign sollte den Trägern Aufschluss geben über Veränderungen oder Nicht-Veränderungen bei den Freiwilligen, während die Wirkungshypothesen Gründe für diese Veränderungen oder NichtVeränderungen aufzeigen und Hinweise auf Stimuli für bestimmte Veränderungen geben können.

\section{Konzeptionelle und theoretische Fundierung}

Mit der oben skizzierten Zielstellung, den Ausgangsthesen und Leitfragen wurde ein partizipativer Entwicklungsprozess der Evaluationsinstrumente initiiert. Der erste Schritt bestand darin, die oben aufgeführten Ausgangsthesen theoretisch zu fundieren. Freiwilligendienste als vielschichtiges soziales Phänomen zwischen individueller Kompetenzentwicklung und Raum gesellschaftlicher Einbettung lassen sich dabei nicht gradlinig auf eine theoretische Basis sondern auf verschiedene theoretische Aspekte zurückführen. Dem Projekt liegt dabei ein konzeptionelles Freiwilligenverständnis als sozialer Lerndienst im jungen Erwachsenenalter (18 bis 27 Jahre) zugrunde. In Abgrenzung zu Freiwilligendiensten der älteren Zielgruppen (Ü27), bei denen andere Ergebnisdimensionen unterstellt und ergänzt werden müssten, wird mit diesem Freiwilligendienstverständnis die biografische Phase der Postadoleszenz mit ihren entsprechenden Entwicklungsaufgaben (Identitätsbildung, sozio-kulturelle Selbständigkeit, Orientierungsaufgaben, Entwicklung von Wert- und Moralvorstellungen etc.) fokussiert (Reinders/Butz 2001). Dem Freiwilligendienst kommt dabei ein aus der soziologischen Lebenslaufforschung und der Entwicklungspsychologie hergeleiteter Moratoriumscharakter zu (Erikson 1988), der dem Individuum Rollenexpe- 
rimente einerseits, Entscheidungs- und Verpflichtungsaufschub im Hinblick auf das Ausbildungs- und Berufsleben andererseits gestattet.

Gleichzeitig wird der Freiwilligendienst konzeptionell verstanden als ein Ort des informellen und non-formalen Lernens. In Anlehnung an Dohmen (2011) liegt dem Projekt und dem Evaluationsinstrument damit ein multidimensionales Bildungsverständnis zugrunde bei dem Lernen als ein ganzheitlicher Prozess und als Veränderung von Wissen, Verhalten, Einstellungen und „Selbst“ verstanden wird. Anders als in schulischen und formalisierten Kontexten wird Lernen dabei als ein Prozess beschrieben, bei dem Handlungs- und Lernprozesse nicht isoliert voneinander, sondern zeitlich und inhaltlich eng verknüpft stattfinden. Dohmen verweist ausdrücklich darauf, dass Lernprozesse dabei $[\ldots]$,nicht nur als bewusste kognitive, sondern auch mehr unbewusste psychische und gefühlsmäßige Verarbeitung von Information verstanden, d.h. als eine ganzheitlich, bewusste und unbewusste, intentionale und beiläufige, theoretische und praktische Verarbeitung von jeder Art von Reizen, Eindrücken, Informationen, Begegnungen, Erlebnissen, Bedrohungen, Anforderungssituationen, symbolische Repräsentation, virtuellen Umwelten etc., die aus der Umwelt auf den Menschen zukommen und von ihm wahrgenommen werden“"(Dohmen 2001: 11).

Vor dem Hintergrund der entwicklungspsychologisch-soziologischen Einordnung und lerntheoretischen Verortung der Freiwilligendienste wurde im nächsten Schritt ein Kompetenzmodell entwickelt, das das oben beschriebene Freiwilligendienstverständnis widerspiegelt. $\mathrm{Zu}$ diesem Zweck wurden verschiedene Kompetenzbegriffe auf ihre Relevanz für die Freiwilligendienste und das Evaluationsinstrument hin untersucht. 


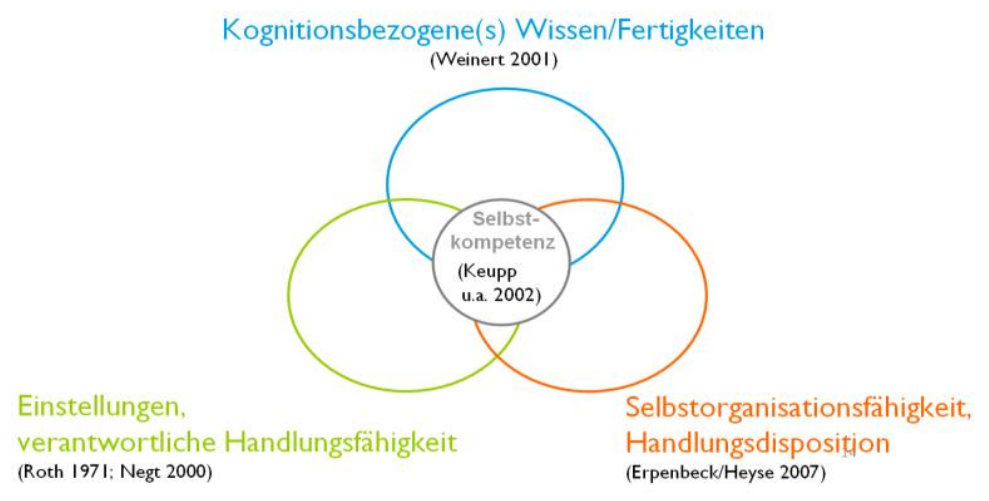

Abb. 2: Kompetenzmodell „Freiwillige“

Quelle: Eigene Darstellung

Im Zentrum dieses Kompetenzmodells steht die Selbstkompetenz im Sinne einer Identitätskompetenz. Im Rückgriff auf Heiner Keupp (Keupp u. a. 2002) fällt darunter die Fähigkeit, in einer mobilen, flexiblen und globalisierten Welt Kohärenz zu schaffen zwischen dem eigenen Entwurf vom Selbst und den Optionen, die die äußere Umwelt zur individuellen Entwicklung bietet. Dabei geht es in einer inneren Dimension um die Fähigkeit, zur „Selbsteinschätzung und Selbstanerkennung“, in einer äußeren Dimension um die „Passungs- und Einbettungsarbeit" in gesellschaftliche Bezüge, um im Ergebnis Authentizität und Sinnhaftigkeit erleben zu können. Freiwilligendienste bieten aus dieser Warte unabhängig von gesellschaftlicher Prägung und sozialer Herkunft den Raum für "Selbstentfaltung und Selbsttätigwerden". Daran anschließend greift das Kompetenzmodell den von Erpenbeck und Heyse (2007) geprägte Kompetenzbegriff als Selbstorganisationsfähigkeit auf und verweist damit auf die freiwilligendienstimmanente Möglichkeit, im Dienst eigene Handlungsdispositionen und Potentiale erkennen, freisetzen und diese flexibel ziel- oder problemlösungsorientiert einsetzen zu können. Die Handlungs- und Problemlösungsfähigkeit wird im Kompetenzmodell wiederum begleitet durch den von Roth (1971) statuierten Kompetenzbegriff als die „Mündigkeit zum verantwortungsvollen Handeln“. Damit ist die Fähigkeit gemeint, das eigene Handeln vor dem Hintergrund ethischer und moralischer Wertvorstellungen reflektieren und in Anlehnung an den Kompetenzbegriff von Oskar Negt (2000) ökologische, technologi- 
sche, soziale und politische Standpunkte im Sinne einer zukunftsfähigen gesellschaftlichen Entwicklung vertreten zu können. Dem von Weinert (2001) geprägten kognitionspsychologischen Kompetenzbegriff entsprechend umfasst das Kompetenzmodell darüber hinaus kognitionsbezogene Fertigkeiten als die Fähigkeit, im Rahmen des Freiwilligendienstes Informationen und Wissen verarbeiten und bedarfsorientiert abrufen zu können.

Auf diesen konzeptionellen und theoretischen Vorüberlegungen beruhend wurden die entsprechenden Ergebnisdimensionen des Freiwilligendienstes als theorie- und hypothesenbasierte Konstrukte für die Befragung festgelegt. Sie umfassen den Bereich der Kompetenzentwicklung (personale Kompetenzen, sozial-kommunikative Kompetenzen, Aktivitäts- und Handlungskompetenz, Diversitäts- und Demokratiekompetenz, Fach- und Sprachkompetenz), den Bereich der Beruflichen Orientierung, der Engagemententwicklung und der Zufriedenheit mit den Prozessen des Freiwilligendienstes. Der Beitrag der Freiwilligendienste zu beruflichen Orientierungsprozessen wird theoretisch hergeleitet anhand der Berufswahltheorien von Bußhoff (1989) und Lange (1976), wonach Berufswahl als ein individueller Entwicklungs- ebenso wie ein Interaktionsprozess zwischen Individuum und Umwelt verstanden wird. Auf den Freiwilligendienst übertragen nehmen Freiwillige den Berufswahltheorien entsprechend ihren Dienst mit individuellen Selbstverwirklichungsinteressen und/oder mit fachlichen Motivationen auf. Während des Freiwilligendienstes erfahren sie in der alltäglichen Interaktion mit VertreterInnen einer Berufsgruppe, im Rahmen ihrer fachlichen Tätigkeiten und in der sozialen Umwelt der Einsatzstelle, ob ihr Selbstkonzept, ihre individuellen beruflichen Interessen und Motive mit der sozialen und fachlichen Umwelt sowie den Anforderungen des Handlungsfeldes kongruent sind oder nicht.

Die theoretische Fundierung der Hypothese, dass Freiwilligendienste einen Beitrag zur Engagementförderung bei den Freiwilligen leisten und das Interesse an bürgerschaftlichem Engagement steigern, beruht auf sozialtheoretischen Überlegungen zur Reziprozität und der Theorie der Gabe von Marcel Mauss (1990) ebenso wie auf sozialkapitaltheoretischen Ausführungen von Bourdieu (1983). Demzufolge bewirkt das Geben/das Schenken (von Zeit, von Engagement) als anthropologische Grundkonstante (Caillé 2008), anders als rein ökonomisch motiviertes Nutzen-Kalkül, immer auch eine Rückkopplung, die neben der persönlich empfundenen sinnhaften Erfahrung 
des Gebens wiederum wechselseitige soziale Beziehungen im Sinne von sozialen Ressourcen und sozialer Einbettung entstehen lässt.

Theoretische Überlegungen zu den Wirkfaktoren von Zufriedenheit in den Freiwilligendiensten lassen sich, der Natur der Sache entsprechend, einerseits auf subjektive Theorien (Beck/Krapp 2006) zurückführen, wenn Freiwillige aus ihrer ganz persönlichen Motivations- und Erwartungsstruktur heraus Prozesse zufrieden oder eher unzufrieden erleben. Auf objektiver Ebene gibt die Zwei-Faktoren-Theorie zur Arbeitszufriedenheit (Herzberg 1968) Aufschluss über arbeitsbezogene inhaltliche Motivationsfaktoren, denen sogenannten Hygienefaktoren (Anerkennung, Entlohnung, Atmosphäre in der Einsatzstelle etc.) gegenübergestellt werden und in der Waagschale beider Faktoren dann Arbeitszufrieden- bzw. -unzufriedenheit konstituieren können.

\section{Operationalisierung: Ergebnisse abbilden}

Mit dem Wunsch, die oben aufgeführten Konstrukte als Ergebnisse von Freiwilligendiensten auf Ebene der Freiwilligen sicht- und messbar zu machen, wurden die theoretisch hergeleiteten Ergebnisdimensionen anhand valider psychometrischer Skalen und Frage-Items aus der Psychologie, der Bildungs- und Evaluationsforschung operationalisiert und PraktikerInnen in einem ersten Fragebogen-Entwurf zur Diskussion gestellt. In einer Folge von Trägerworkshops und unter wissenschaftlicher Beratung zu methodischen Aspekten der Fragebogenentwicklung wurde das Evaluationsinstrument im Rahmen eines kognitiven Pre-Tests mit anschließender Fokusgruppendiskussion und eines inhaltlichen Pre-Tests im Online-Verfahren getestet. Auf der Basis erster statistischer Überprüfungen der Befragungsergebnisse wurden Frage-Items zusammengefasst bzw. reduziert und Strukturen oder auffällige Muster im Antwortverhalten identifiziert.

Im Ergebnis des Projekts steht damit ein Evaluationsinstrument für die Ergebnisqualität von Jugendfreiwilligendiensten. Begleitet von einem Handbuch für die Nutzung und Auswertung des Fragebogens erlaubt der Fragebogen allen Trägern, die Ergebnisse ihrer Freiwilligendienste zunächst systematisch zu messen und - wenn gewollt - auch zu steuern. Anhand statistischer Kennwerte gibt der Fragebogen zunächst quantitative Informationen und damit Hinweise, denen man sich auf qualitativen Wegen (Fokus- 
gruppendiskussionen, problem- oder themenzentrierten Interviews etc.) weiter annähern kann. Bezogen auf jedes im Fragebogen abgebildete Konstrukt bieten ergänzend zum Fragebogen entwickelte Wirkungshypothesen, vom ermittelten Ergebnis ausgehende Hinweise auf träger- oder einsatzstellenspezifische Prozesse und Strukturen, die als Stellschrauben von Qualität Veränderungen und damit Ergebnisse stimulieren können.

Methodisch bleibt aufgrund der Zuordnungslücke die Herausforderung, einzelne (Nicht-)Veränderungen bei den Freiwilligen auf Prozesse und/oder Strukturen der Träger zurückzuführen. Dieses methodische Manko wurde auch im Rahmen des Fachtags ausführlich diskutiert. Um die methodische Lücke zu schließen, bedarf es einer Vergleichsgruppe, die Aufschluss darüber gibt, welche Veränderungen direkt im Ergebnis des Freiwilligendienstes stehen. Der Fragebogen vermag jedoch Hinweise auf potentielle Strukturund Prozessverbesserungen zu geben, dort wo mehrere Freiwillige unterschiedliche Ergebnisse erzielen.

\section{Chancen und Grenzen des Befragungsinstruments}

Quifd hat sich mit diesem Projekt der Ergebniserfassung und damit der Qualitätsentwicklung von Freiwilligendiensten ein weiteres Stück genähert. Was Quifd nicht leisten konnte, ist, im Rahmen der Projektentwicklung alle methodischen Hürden bzw. anlässlich des Fachtags alle kritischen Vorbehalte gegen Zahlen und Kennwerte oder die Befürchtungen der Instrumentalisierung des Fragebogens auszuräumen. Auch steht ein echter Längsschnittvergleich (Vorher- und Nachher-Befragung einer Person) noch aus, der Hinweise auf mögliche weitere Verbesserungen des Fragebogens geben könnte. Der Fragebogen kann jedoch, so die Anregung von Quifd, flexibel eingesetzt werden und in trägerspezifische Fragemodule integriert werden, um so Erkenntnisse für die eigene Qualitätsarbeit zu generieren.

Analog zu den bestehenden Standards für Struktur- und Prozessqualität stellen zwei Qualitätsstandards für die Ergebnisqualität als Kann-Kriterien einen Anreiz dar, die Ergebnisqualität im Rahmen einer Selbstevaluation zu erheben und durch die externen Quifd-GutachterInnen prüfen zu lassen. Erst eine höhere Datenmenge und die Rückkopplung der Trägerorganisationen wird Quifd dazu befähigen, den Fragebogen zu justieren und die Auswertungsmatrix zu konturisieren. Eine angepasste Nutzung wird hier aber auch 
verstanden als die Möglichkeit, den Fragebogen als Grundlage für ergänzende qualitative und multimethodische Erhebungen, ja, die Ergebnisse nicht nur als Gegenstand eines Qualitätsstandards, sondern als Hinweis und Indikator zu nutzen, um darüber mit den am Freiwilligendienst und dessen Qualität beteiligten Akteuren ins Gespräch zu kommen. Der Fragebogen steht inklusive des Handbuchs auf Anfrage bei Quifd (www.quifd.de; kontakt@quifd.de) gegen eine einmalige Schutzgebühr von 30 Euro für zertifizierte bzw. 50 Euro für nicht-zertifizierte Trägerorganisationen allen Interessierten zur Verfügung.

\section{Der Fachtag: Rezeption des Befragungsinstruments}

Die Möglichkeiten und Begrenzungen eines Fragebogens als Abbild der freiwilligenbezogenen Ergebnisse, wurden auch im Rahmen des von Quifd durchgeführten Fachtags „, Ergebnisqualität von Freiwilligendiensten erkennen und optimieren“ am 9. September 2013 in Berlin kritisch diskutiert. Der Fachtag veranschaulichte einerseits die oben dargestellte Kontroverse über das Verständnis der Freiwilligendienste zwischen Moratorium und (strukturiertem) Lernort, andererseits die Ambivalenz zwischen intrinsischem Anliegen der Trägerorganisationen, Ergebnisse nachvollziehbar zu machen und der Befürchtung vor Verzweckung und externer Kontrolle der Freiwilligendienste. So wurde der Fragebogen von den einen als methodische Unterstützung und Mehrwert gesehen, während die anderen den Fragebogen als Kontrollinstrument und statistisches Zahlenwerk bewerteten. Der Fragebogen, so eine Befürchtung, könne im Sinne förderpolitischer Steuerung oder der Festschreibung von Kompetenzen für den Arbeitsmarkt instrumentalisiert werden und der Verzweckung der Dienste Vorschub leisten. In der auf eine Podiumsdiskussion folgenden Kleingruppendiskussion konnten Ansatzpunkte identifiziert werden, um diesen Kritikpunkten zu begegnen. So sollte der Fragebogen auf die individuellen Bedürfnisse der Träger angepasst und damit eine generalisierende und vergleichende Bewertung der Trägerorganisationen vermieden werden. Darüber hinaus wurde betont, dass es im Anhang des Instruments wichtig sei, stets darauf zu verweisen, dass der Fragebogen nur einen Ausschnitt der Kompetenzen erfassen kann und dabei ausschließlich die Selbsteinschätzung des Freiwilligen misst. Abschließend lässt sich festhalten, dass die Haltungen der verschiedenen Akteure zur Ergebnismessung durch Längsschnittbefragungen höchst divers und längst nicht 
alle Widersprüche aufgehoben sind. Dabei sind es sicherlich nicht die erhobenen Zahlenwerte an sich, die Aufschluss geben oder Scheinobjektivität vermitteln sollen, sondern die Freiwilligen, die bei allem Raum für Unverzwecktheit und unter Anerkennung der vielschichtigen, höchst subjektiven Erfahrungen und Ergebnisse einen Anspruch haben auf Einsatzstellen, in denen sie u. a. Selbstwirksamkeit empfinden, sich weiterentwickeln und sich auf fachliche und persönliche BegleiterInnen verlassen können. Und damit sind es die Freiwilligen, die einen Anspruch auf qualitätvolle Strukturen, Prozesse und Ergebnisse haben und mittels des hier vorgestellten Befragungsinstruments zumindest einen ersten Aufschluss darüber geben können.

\section{Literaturverzeichnis}

Beck, Klaus; Krapp, Andreas (2006): Wissenschaftstheoretische Grundfragen der Pädagogischen Psychologie, in: Pädagogische Psychologie. Weinheim [u. a.], hrsg. von A. Krapp und B. Weidenmann, S. 33-72.

Bourdieu, Pierre (1983): Ökonomisches Kapital, kulturelles Kapital, Soziales Kapital, in: Soziale Ungleichheiten. Sonderband 2 der Sozialen Welt, hrsg. von Reinhard Kreckel, Göttingen, S. 183-198.

Bußhoff, Ludger (1989): Berufswahl. Theorien und ihre Bedeutung für die Praxis der Berufsberatung. 2., neubearb. Aufl., Stuttgart u. a.

Caillé, Allain (2005): Anthropologie der Gabe, Frankfurt/M.-New York.

Dohmen, Günther (2001): Das informelle Lernen - Die internationale Erschließung einer bisher vernachlässigten Grundform menschlichen Lernens für das lebenslange Lernen aller, Bonn.

Erikson, Erik (1988): Der vollständige Lebenszyklus. 2. Aufl. 1992, Frankfurt a.M.

Erpenbeck, John und Volker Heyse (Hrsg.) (2007): Kompetenzmanagement. Methoden, Vorgehen. $K O D E$. ${ }^{\circledR}$ und $K O D E \circledast X$ im Praxistest, Münster / New York.

Herzberg, Frederick (2003): Was Mitarbeiter in Schwung bringt, in: Harvard Business Manager, Heft 04/2003, S.50-62.

Keupp, Heiner u. a. (2002): Identitätskonstruktionen. Das Patchwork der Identitäten in der Spätmoderne, Hamburg.

Lange, Elmar (1976): Berufswahl als Interaktionsprozess. Theoretische Vorüberlegungen für ein empirisches Projekt, in: Kölner Zeitschrift für Soziologie und Sozialpsychologie 1976(28), S. 479-505.

Mauss, Marcel (1990): Die Gabe - Form und Funktion des Austauschs in archaischen Gesellschaften, Frankfurt/M.

Negt, Oskar (1998): Lernen in einer Welt gesellschaftlicher Umbrüche, in: Lernkonzepte im Wandel. Die Zukunft der Bildung, hrsg. von Heinrich Dieckmann und Bernd Schachtsiek, Stuttgart, S. 21-44.

Reinders, Heinz und Petra Butz (2001): Entwicklungswege Jugendlicher zwischen Transition und Moratorium, in: Zeitschrift für Pädagogik 47 (2001) 6, S. 913-928.

Weinert, Franz E. (2001): Vergleichende Leistungsmessung in Schulen - Eine umstrittene Selbstverständlichkeit, in: Leistungsmessungen in Schulen, hrsg. von Franz E. Weinert, WeinheimBasel, S. $27 \mathrm{f}$.

Roth, Heinrich (1971): Pädagogische Anthropologie, Bd. 2. Hannover. 


\begin{abstract}
Ute Elisabeth Volkmann

Making Quality visible: Outcome Quality and Evaluation Tools for Volunteer Services

Volunteer Services, considered as individual educational services, are discussed controversially in terms of their immediate results and long-term impacts. Quifd, the Agency for Quality in Volunteer Services with its particular objective of quality development, has taken on the controversial interest in outcome-oriented evaluation of volunteer services in the project "Development of Standards for the Quality of Results and of Evaluation Tools for Volunteer Services". With the overall objective to support volunteer service organisations in their quality development efforts, theory-based evaluation tools for longitudinal studies, outcome assumptions and functional chains as well as standards for the quality of results were developed. In conflict between a general openness for any kind of personal development and standardized results on the volunteer's side, the questionnaire serves as a first "display" of individual and aggregated changes and results. Moreover the project aims at identifying structures and procedures determining the quality of results and aims at fostering innovative practical approaches for quality development. The project results were presented during the conference: "Quality of Results in Voluntary Services " on the 9th of September in Berlin. Representatives from politics, science and practitioners discussed the results of the project as well as the chances and limits of result measurement. This paper presents the outcomes of the project and the discussion during the conference.
\end{abstract}

Keywords: Quality Development, Quality of Results, Impact-expectation, Comprehensive Evaluation Tool, Standards for the Quality of Results, MicrolevelEffectiveness, Outcome-Assumptions 